\title{
Real-time fractional order system identification using recursive least squares method
}

\author{
Walid TOUIL ${ }^{(1)^{*}}$, Samir LADACl ${ }^{(1,2)}$, Abdelhafid CHAABI ${ }^{(3)}$ \\ (1) SP-Lab Laboratory, Depart. of Electronics, University of Mentouri Brothers \\ Route de Ain Elbey, Constantine 25000, Algeria \\ (2) dept of EEA, National polytechnic School of Constantine, \\ BP. 75 A, Ali Mendjli, Constantine 25100, Algeria \\ (3) dept. of Electronics, University of Mentouri Brothers Constantine 1, \\ Route de Ain Elbey, Constantine 25000, Algeria \\ "Corresponding author Email: walid25touil@gmail.com
}

\begin{abstract}
In this paper, we address the problem of fractional order systems real-time identification based on recursive least squares technique. The fractional order model is approximated using the Charef Singularity function method and Grünwald numerical approximation for fractional order integral and derivative. We show by numerical simulation example that the identified model represents the original system efficiently.
\end{abstract}

Keywords: fractional order system; identification system; recursive least squares (RLS) technique; Charef approximation method; Grünwald numerical approximation.

\section{INTRODUCTION}

The study of fractional order systems has been receiving considerable attention due to the fact that many physical systems are well characterized by these models [1]. Many examples of such systems can be cited like energy systems [2], electrical machines [3], chemical systems [4], etc.

The need for effective identification of fractional order systems is motivated by control objectives that necessitate a precise and accurate model [5]. Many methods such as out-put error, least square and instrumental variable are widely used for realtime parameter identification [6].

Many researchers have contributed to fractional order systems identification art. Oustaloup [7] proposed a method using a discretized fractional differential equation, and in another pioneering work, Trigeassou [8] proposed a technique based on the approximation of integrals using a modal approach. Djouambi at al. [9] proposed an identification technique providing recursive parameters estimation of fractional order models, where the identification model is defined by a generalized ARX structure obtained by discretization of a continuous fractional order differential equation. More recently, Idiou et al. [10] proposed an adjustable fractional order differentiator for this aim. Li et al. [11] proposed a parameter identification method for fractional order nonlinear systems using the Haar wavelets to represent the input and output signals, and then convert the nonlinear differential into a corresponding integral equation. Gao [12] developed a modulating function-based system identification method. Also, many works addressed the fractional order nonlinear systems identification problem [13]. Some authors have used fractional order models to identify real processes and phenomena like thermal dynamics of buildings [14-15].

In this paper, we propose a simple approach for identifying fractional order systems defined by their fractional order differential equation using the RLS with forgetting factor technique. We show by a numerical example that we are able to find out an efficient model for the system representation.

This paper is organized as follows: Section 2 presents some mathematical basics of fractional calculus and the considered approximation approach for fractional order functions. Section 3 presents a detailed and description of the fractional integration operator, the singularity function method of Charef. Section 4 presents the recursive least square (RLS) with forgetting factor algorithm used to estimate the parameters of the fractional order differential equation. A simulation example is given in Section 5 . Finally, conclusions are given in Section 6.

\section{FRACTIONAL ORDER OPERATORS}

There exists many formulations for the fractional order integration definition; the 
most popular are those of Grünwold-Letnikov (GL), Riemann-Liouville (RL) and Caputo [16].

Riemann-Liouville (RL) fractional order integral is expressed as:

$$
I_{n} f((t))=\frac{1}{\Gamma(n)} \int_{0}^{t}(t-\tau)^{n-1}
$$

when $\Gamma(\mathrm{n})$ is the gamma function

$$
\Gamma(n)=\int_{0}^{\infty} x^{n-1} e^{-x} d x
$$

If $I_{n}(f(t))$ is convolution of function with the impulse response:

$$
h_{n}(t)=\frac{t^{n-1}}{\Gamma(n)}
$$

On the fractional operator whose Laplace transform is

$$
I_{n}(s)=L\left\{h_{n}(t)\right\}=\frac{1}{s^{n}}
$$

Notice that in the integer order case $(n=1)$, the integral is characterized by $h 1(t)=H(t)$ (unit step function or Heaviside function:

$$
I_{1}(s)=L\left\{h_{1}(t)\right\}=\frac{1}{s}
$$

Fractional differentiation is the dual operation of fractional integration.

$$
x(t)=I_{n}(v(t)) \text { or } X(s)=\frac{1}{s^{n}} V(t)
$$

Reciprocally, $v(t)$ is the $n^{\text {th }}$ order fractional derivative of $\mathrm{x}(\mathrm{t})$ defined as :

$$
v(t)=D_{n}(x(t)) \text { or } V(s)=s^{n} X(t)
$$

Where $D_{n}(s)=s^{n}$ represents the Laplace transform of the fractional differentiation operator (for initial conditions equal to zero). This fractional derivative definition is based on the operator $\mathrm{I}_{\mathrm{n}}(\mathrm{s})$, without analytical formulation of $D_{n}(x(t))$ : it is the implicit definition of the fractional derivative.

\section{APPROXIMATION METHOD OF THE FRACTIONAL INTEGRATOR OPERATOR}

This approximation method called singularity function algorithm proposed by Charef allowing us to convert the fractional calculus to rational one [17].

\section{G. Fractinal order integration}

The fractional order integrator is represented by the following irrational transfer function.

$$
H(s)=\frac{1}{s^{\beta}}, 0<\beta<1
$$

The singularities (poles, zeros), are computed using following algorithms:

$$
\begin{gathered}
P_{i}=(a b)^{i} P_{0} \quad i=0,1, \ldots, N \\
Z_{i}=(a b)^{i} a P_{0} \quad i=0,1, \ldots N-1
\end{gathered}
$$

The approximation analog transfer function is calculated by:

$$
H(s)=\frac{K_{I}}{\left(1+\frac{s}{w_{c}}\right)^{\beta}}=K_{I} \frac{\prod_{i=0}^{N-1}\left(1+\frac{s}{Z_{i}}\right)}{\prod_{i=0}^{N}\left(\left(1+\frac{s}{P_{i}}\right)\right.}
$$

Where $\mathrm{a}, \mathrm{b}, \mathrm{K}_{\mathrm{I}}, \mathrm{P}_{0}, \mathrm{Z}_{0}$ are given by:

$$
\begin{gathered}
a=10^{\frac{\varepsilon}{10(1-\beta)}} \\
b=10^{\frac{\varepsilon}{10 \beta}} \\
P_{0}=\omega_{c} \sqrt{b} \\
Z_{0}=a P_{0} \\
K_{I}=\frac{1}{w_{c}^{\beta}} \\
\omega_{c}=\omega_{b} \sqrt{10^{\frac{\varepsilon}{10 \beta}}-1}
\end{gathered}
$$

$\varepsilon$ is the tolerated error in $\mathrm{dB}$

The approximation order is $\mathrm{N}$ is calculated by fixing the working frequency bandwidth $\omega \in\left[\omega_{b}, w_{h}\right]$ such that $\omega \gg \omega_{c}$

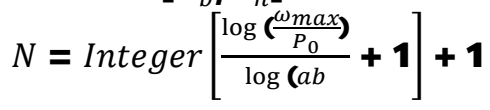

$H$. Example of Sinularity function approximation We considerate system:

$$
\mathrm{G}=\frac{1}{(1+\mathrm{t} * \mathrm{~s})^{\mathrm{m}}}
$$

Where $\mathrm{t}=1$ and $\mathrm{m}=0.6$. Using the approximation method of Charef with the specifications:

$w b=0.01$; $w h=100$; we obtain a rational transfer function and draw the comparative bode response with that of the original fractional order transfer (19), given in fig. 1.
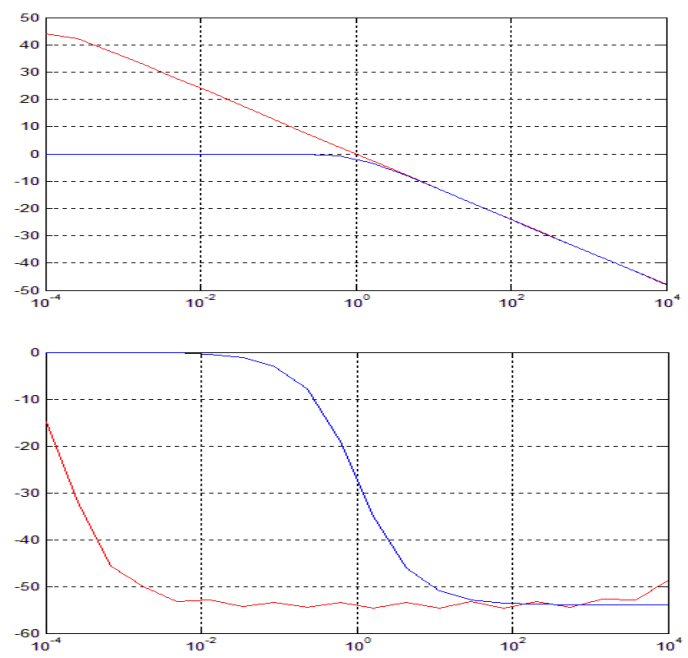

Fig.1. Bode plot for the fractional system and its approximation. 
It is obvious that at the considered frequency bandwidth, the amplitude and the phase of the approximating function correspond to those of the fractional order system.

\section{IDENTIFICATION PROCEDURE}

The recursive least squares method (RLS) [18-19] is a popular method of for modeling in industrial application, it can be applied to identification using linear model with respect to parameters (Lp) model given by,

$$
\hat{y}(\mathrm{t})=\varphi(\mathrm{t})^{\mathrm{T}} \hat{\theta}(\mathrm{t})
$$

where $\varphi(\mathrm{t}), \hat{\theta}$ and $\hat{\mathrm{y}}(\mathrm{t})$ are regression vector, estimated parameters and estimated measure respectively. By minimizing the quadratic criterion function $\mathrm{J}$, estimation of, can be obtained,

$$
\mathrm{J}(\hat{\theta}, \mathrm{t})=\sum_{\mathrm{i}=1}^{\mathrm{t}}\left[\mathrm{y}(\mathrm{i})-\varphi(\mathrm{i})^{\mathrm{T}} \hat{\theta}(\mathrm{i})\right]^{2}
$$

Solving for the minimizing parameters, we get the closed form solution as follows:

$$
\hat{\theta}(\mathrm{t})=\left[\sum_{\mathrm{i}=1}^{\mathrm{t}} \varphi(\mathrm{i}) \varphi(\mathrm{i})^{\mathrm{T}}\right]^{-1} \sum_{\mathrm{i}=1}^{\mathrm{t}} \varphi(\mathrm{i}) \mathrm{y}(\mathrm{i})
$$
where $\mathrm{y}(\mathrm{i})$ represents the measure Alternately, The recursive least squares square (RLS) handles with algorithm for recursively estimating parameters in linear regression models. Most of the time, we are interested in realtime parameter estimation. Therefore, it is computationally more efficient if we update the estimates in equation (22) recursively as new data becomes available online. The recursive form is given by:

where

$$
\begin{gathered}
\hat{\theta}(\mathrm{t})=\hat{\theta}(\mathrm{t}-1)+\mathrm{p}(\mathrm{t}) \varphi(\mathrm{t})[\mathrm{y}(\mathrm{t})- \\
\left.\varphi^{\mathrm{T}}(\mathrm{t}) \hat{\theta}(\mathrm{t}-1)\right]
\end{gathered}
$$

$$
\mathrm{p}(\mathrm{t})=\left[\sum_{\mathrm{i}=1}^{\mathrm{t}} \varphi(\mathrm{i}) \varphi(\mathrm{i})^{\mathrm{T}}\right]^{-1}
$$

Note that the term $\mathrm{y}(\mathrm{t})-\varphi^{\mathrm{T}}(\mathrm{t}) \hat{\theta}(\mathrm{t}-1)$ is the prediction error where $\varphi^{\mathrm{T}}(\mathrm{t}) \hat{\theta}(\mathrm{t}-1)$ is the prediction of $\mathrm{y}(\mathrm{t})$ using model variable at time $\mathrm{t}-1$

\section{A. RLS with forgeting factor}

In the least square method, forgetting can be viewed as giving less weight to older data and more weight to recent data. The lossfunction is then defined as follows:

$$
\mathrm{J}(\hat{\theta}, \mathrm{t})=\sum_{\mathrm{i}=1}^{\mathrm{t}} \lambda^{\mathrm{t}-\mathrm{i}}\left[\mathrm{y}(\mathrm{i})-\varphi(\mathrm{i})^{\mathrm{T}} \hat{\theta}\right]^{2}
$$

Where $\lambda$ is called forgetting factor $0<\lambda \leq 1$ The RLS algorithm with forgetting factor we need to implement are:

$$
\begin{gathered}
\hat{\theta}(\mathrm{t})=\hat{\theta}(\mathrm{t}-1)+\mathrm{K}(\mathrm{t})\left[\mathrm{y}(\mathrm{t})-\varphi^{\mathrm{T}}(\mathrm{t}) \hat{\theta}(\mathrm{t}-\right. \\
1)] \\
\mathrm{K}(\mathrm{t})=\mathrm{P}(\mathrm{t}) \varphi(\mathrm{t})=\frac{\mathrm{p}(\mathrm{t}-1) \varphi(\mathrm{t})}{\left[\lambda+\varphi^{\mathrm{T}}(\mathrm{t}) \mathrm{P}(\mathrm{t}-1) \varphi(\mathrm{t})\right]} \\
\mathrm{P}(\mathrm{t})=\frac{(27)}{\lambda}
\end{gathered}
$$

When $\lambda=1$ this is simply the RLS algorithm.

In order to perform the recursion, we need an initial value for $P(0)$ and initial estimate for $\varphi(0)$, we assume $P(0)=I$ and $\varphi(0)=0$

Using algorithm with a forgetting factor we may speak about real time identification.

\section{IDENTIFICATION EXAMPLE}

In this part, we using the least square technique to estimate the parameters of a fractional order system given by its differential equation,

$$
\sum_{l=0}^{L} a_{l} D^{\alpha_{l}} y(t)=\sum_{m=0}^{M} b_{m} D^{\beta_{m}} u(t)
$$

Where the definition of Grünwald-Leitnikov is considered for the approximation of the fractional order integrals and derivatives.

The parameters al and bm represent the poles $p(i)$ and zeroes $z(i)$ of the approximated transfer function by Charef's approach.

For $\mathrm{N}=10$, we obtain the following Fig. 2 and 3 for the comparative output behavior and the parameters estimation respectively.

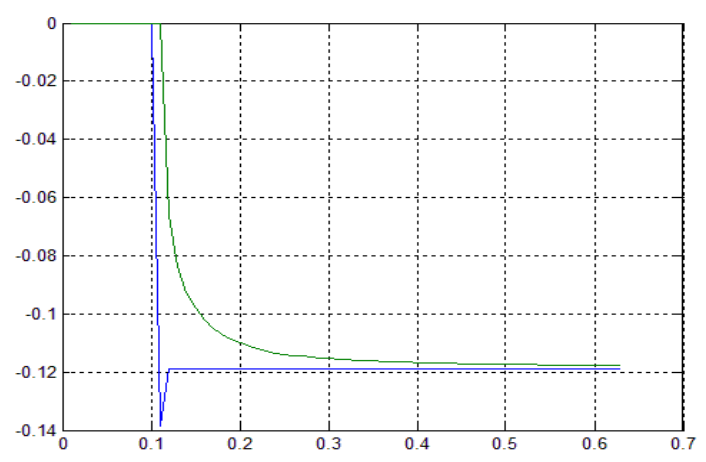

Fig. 2. Comparative responses of the fractional order system (in blue) and the estimated model (in green).

We remark that the responses of the original fractional order system and the identified model are very close and converge to the same steady-state value, with a small 
difference in the transient phase. Fig. 3 shows that the parameters converge uniformly to their final values.

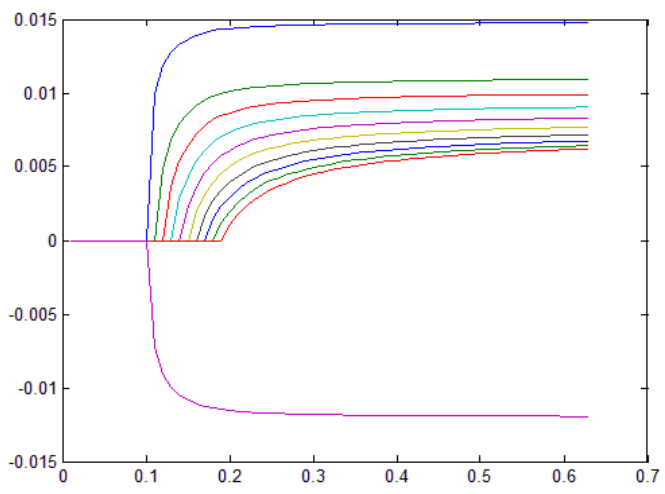

Fig. 3. Parameter estimation.

\section{CONCLUSION}

The identification of fractional order systems is very important with regard to potential applications in control of processes. In this paper we consider the fractional order systems modeling and identification by recursive least square (RLS) with forgetting factor technique using the Singularity Function approximation method for fractional order integrators. The simulation example shows that the RLS technique combined with the approximation of fractional order system is able to deliver a good model. Research work is continuing toward the design of new identification algorithms for more accuracy and efficiency.

\section{References}

[1] T.T. Hartley, and C.F. Lorenzo, "Fractionalorder system identification based on continuous order-distributions". Signal Processing, vol. 83, no. 11, pp. 2287-2300, 2003.

[2] J.L. Battaglia, O. Cois, L. Puigsegur, and A. Oustaloup, "Solving an inverse heat conduction problem using a non integer identified model", International Journal of Heat and Mass Transfer 44, 2671-2680, 2001.

[3] A. Jelloul, K. Jelassi, J.C. Trigeassou, and P. Melchior, "Comparison of Fractional Identification Techniques for Rotor Skin Effect in Induction Machines", IJCSI, Vol. 8, Issue 3, 2011.

[4] G. Garcia, and J. Bernussou, "Identification of the dynamics of a lead acid battery by a diffusive model", Proceedings of ESSAIM 5, 1998.

[5] S. Ladaci, and A. Charef, On Fractional Adaptive Control, Nonlinear Dynamics, vol. 43, no. 4, pp. 365-378, 2006.
[6] A. Khadrahoui, K. Jelassi, J.C. Trigeassou. Identification of a fractional order model by a least squares technique: $\mathrm{Hn}$ model. Progress in Computing Applications, Vol. 2, No. 2, September 2013.

[7] A.Oustaloup, L. Le Lay, and B. Mathieu, "Identification of non-integer order system in the time-domain". In IEEE-CESA'96, SMC IMACS Multiconference, 1996.

[8] J.-C. Trigeassou, T. Poinot, J. Lin, A. Oustaloup,. and F. Levron, "Modeling and identification of a non-integer order system". In Proc. Of the European Control Conference, 1999.

[9] A. Djouambi, A. Voda, and A. Charef, "Recursive prediction error identification of fractional order models", Communications in Nonlinear Science and Numerical Simulation, vol. 17, no. 6, pp. 2517-2524, 2012.

[10] D. Idiou, A. Charef, and A. Djouambi, "Linear fractional order system identification using adjustable fractional order differentiator", IET Signal Processing, vol. 8, no. 4 , pp. 398-409, June 2014.

[11] Y. Li, M. Jiang, and J. Li "Parameter Identification of Fractional Order Nonlinear System Based on Haar Wavelet Operational Matrix". Proceedings of the 2017 International Conference on Applied Mathematics, Modeling and Simulation (AMMS 2017).

[12] Z. Gao, "Modulating function-based system identification for a fractional-order system with a time delay involving measurement noise using least-squares method", International Journal of Systems Science, vol. 48, no. 7, 2017.

[13] M.-R. Rahmani, and M. Farrokhi, "Nonlinear dynamic system identification using neurofractional-order Hammerstein model", Transactions of the Institute of Measurement and Control, vol. 40, no. 13, pp. 3872-3883, 2018.

[14] L. Chen, B. Basu, and D. McCabe, "Fractional order models for system identification of thermal dynamics of buildings", Energy and Buildings, vol. 133, pp. 381-388, 2016.

[15] P. Shah, S. Agashe, and V. Vyawahare, "System Identification with Fractional-order Models: A Comparative Study with Different Model Structures", Progr. Fract. Differ. Appl. Vol. 4, no. 4, pp. 533-552, 2018.

[16] K.S. Miller, and B. Ross. "An Introduction to the Fractional Calculus and Fractional Differential Equations". Wiley, New York, 1993.

[17] A. Charef, H.H. Sun, Y.Y. Tsao, and B. Onaral, "Fractal system as represented by singularity function", IEEE Transactions on Automatic Control, vol. 37, no. 9, pp. 14651470, 1992.

[18] K.J. Åström, B. Wittenmark. Adaptive Cntrol. Second edition, Addison-Wesley Publishing Company, 1995.

[19] S. Ladaci, S. Cheniki, H. Bendjama, and A. Charef, "Indirect Fractional Adaptive Pole Placement Control", In the 3rd International Conference on Control, Engineering \& Information Technology, CEIT'2015, Tlemcen, Algeria, 25--27 May 2015. 